\title{
Compressed Sensing Maximum Likelihood Channel Estimation for Ultra-Wideband Impulse Radio
}

\author{
Ted C.-K. Liu, Xiaodai Dong and Wu-Sheng Lu \\ Department of Electrical and Computer Engineering, University of Victoria, Victoria, BC, Canada \\ e-mail: $\{$ tcliu, xdong,wslu\}@ece.uvic.ca
}

\begin{abstract}
One of the most attractive features of ultrawideband impulse radio is the collection of rich multipath with the transmission of ultra-short pulses. Exploiting the rich multipath diversity with channel estimating Rake receivers enables significant energy capture, higher performance and flexibility than suboptimal receivers. Although data-aided (DA) maximum likelihood (ML) channel estimator shows a promising performance, its implementation is restricted by the Nyquist sampling criterion. The emerging theory of compressed sensing (CS) describes a novel framework to jointly compress and detect a sparse signal with fewer samples than the traditional Nyquist criterion. In this paper, we propose a CS-ML channel estimator which combines the compression framework of $\mathrm{CS}$ for sampling rate reduction while retaining the noise statistics formulation of ML to achieve a reliable performance. Simulation assessment indicates that, with far fewer measurements, the performance of our proposed scheme supersedes that of the $\ell_{1}$-norm minimization estimator of $\mathrm{CS}$ and can be as close as the ML, but with a reduction in complexity.
\end{abstract}

\section{INTRODUCTION}

The rich multipath characteristic of a wireless communication system operating with sub-nanosecond pulses is one of the most attractive features of ultra-wideband (UWB) impulse radio (IR) [1]. Coupled with the fine time resolution and lowpower implementation, UWB-IR is an emerging candidate for both localization and ranging applications. The rich multipath diversity of an impulsive wideband channel calls for the use of Rake receivers for significant energy capture, higher performance and flexibility, despite its complexity over suboptimal counterparts [2]. In addition, accurate multipath delays (and amplitudes) estimation is indispensable for precision asset localization and tracking with UWB-IR. Maximum likelihood (ML) channel estimator for UWB was first proposed in [3] for the transmission of an isolated pulse. Its data-aided (DA) alternative was later suggested in [4] to enhance the estimator accuracy, especially in a noisy environment. Although the ML scheme is shown to be a superior estimator, the tremendous bandwidth of UWB signal renders its implementation difficult because of the Nyquist criterion.

Most existing literatures on ML complexity reduction tackle the issue either by redefining the problem or eliminating the use of ML altogether. Refs. [5] and [6] approached the complexity issue by way of formulating it as either a frame synchronization or least squares timing recovery problem, respectively. In [2], the ML estimator was simplified by recognizing multipaths do arrive in clusters, and searching only for rays falling into the highest energy clusters. Although they are of low-complexity, their performance implicitly depends on acquiring high-rate samples. Ref. [7] proposed a finite rate of innovation approach which projects a signal into lower dimensional subspace. Unfortunately, due to the closely spaced path arrivals inherent in UWB-IR, the solution to rate of innovation is often ill-conditioned. Despite these attempts, the most critical issue - Nyquist sampling rate reduction - of the ML channel estimator has yet to be addressed.

The emerging theory of compressed sensing (CS) outlines a novel strategy to jointly compress and detect a sparse signal with fewer sampling resources than the traditional method. For a signal $\mathbf{x} \in \mathbb{R}^{N}$ which is $Y$ sparse, with $Y \ll N$ being an integer, compressed sensing shows that with high probability $\mathrm{x}$ can be reconstructed from $M$ compressive measurements when $M \geq C Y \log (N / Y) \ll N$, where $C \geq 1$ is the oversampling factor [8], [9]. Compressed sensing for UWB was first proposed in [10] as a generalized likelihood ratio test receiver taking advantage of the signal structure by incorporating pilot assisted modulation. It was later discussed in [11] as an alternative for UWB channel estimation. Both cases estimate the signal structure via the matching pursuit (MP) $\ell_{1}$-norm minimization algorithm. Unfortunately, how well MP estimates ties directly to the design parameters, such as the number of iterations and residual error for convergence [10], [11], which are subject to change depending on the environment. In addition, MP requires a complete $N \times N$ orthonormal basis as the dictionary for accurate estimation and is ineffective when there is noise present.

To alleviate the burden of acquiring Nyquist-rate samples, we propose a compressed sensing maximum likelihood (CSML) channel estimator that adopts the compression framework of CS for sampling rate reduction while retaining the noise statistics formulation of ML to achieve a reliable performance. Our approach negates the subjective use of MP design parameters. Simulation assessment indicates that the CS-ML supersedes MP in performance, especially in the low signal-tonoise ratio (SNR) environment where the CS-ML can transmit multiple pilot symbols to boost the detection probability. Additionally, CS-ML is shown to achieve performance equal to $\mathrm{ML}$, but with a reduction in complexity.

The rest of the paper is organized as follows: Section II describes our system model, including the derivation of CSML, Section III outlines the implementation issues, Section IV presents the simulation results and concluding remarks are given in Section $\mathrm{V}$ 


\section{System Model}

We consider a single-user communication system that sends a series of $K$ pilot symbols for channel estimation prior to data transmission. The received signal can be modeled as

$$
y(t)=\sum_{k=0}^{K-1} b_{k} \sum_{j=0}^{N_{f}-1} \sum_{l=1}^{L} \alpha_{l} s\left(t-\tau_{l}-j T_{f}-k T_{s}\right)+n(t),
$$

where $\left\{b_{k}\right\}$ are the pilot symbols taking values $\pm 1, \alpha_{l}$ and $\tau_{l}$ are the amplitude and delay of the $l$-th multipath, respectively, $s(t)$ is the received Gaussian pulse of duration $T_{p}$, and $T_{f}$ is the frame time. There are $N_{f}$ frames per symbol for a symbol period of $T_{s}=N_{f} T_{f}$ and $n(t)$ is the additive white Gaussian noise (AWGN) with double-sided power spectral density $N_{0} / 2$. The pilot symbols are transmitted over an observation interval of $T_{0}=K T_{s}$ seconds in which the dense multipath channel is assumed to be static. In (1), we model the channel as an $L$-tap tapped delay line with a maximum delay spread $T_{m d s}$ seconds, and we assume $T_{f}>T_{m d s}$ so there is no inter-frame interference. Without loss of generality and to simplify the notation, we let the polarity of all pilot symbols to be 1 and consider only a single frame, i.e., $N_{f}=1$.

Given the signal in (1), we model the parameters $\boldsymbol{\alpha}=$ $\left[\alpha_{1}, \alpha_{2}, \ldots, \alpha_{L}\right]$ and $\boldsymbol{\tau}=\left[\tau_{1}, \tau_{2}, \ldots, \tau_{L}\right]$ as unknown but deterministic quantities. Let $\boldsymbol{\psi}=(\boldsymbol{\alpha}, \boldsymbol{\tau})$ be the continuoustime channel parameter, we apply compressed sensing by expanding (1) with $M$ orthonormal basis functions as

$$
\begin{aligned}
y(t) & =\sum_{k=0}^{K-1} \sum_{m=1}^{M} \breve{y}_{k, m} \phi_{m}\left(t-k T_{s}\right), \\
\breve{y}_{k, m} & :=\int_{k T_{s}}^{(k+1) T_{s}} y(t) \phi_{m}(t) \mathrm{d} t, \quad m=1, \ldots, M \\
& =\breve{s}_{k, m}+\breve{n}_{k, m}, \quad m=1, \ldots, M,
\end{aligned}
$$

where $\breve{y}_{k, m}$ is the projection coefficient of the received signal to the $m$-th basis in the $k$-th symbol. Similarly, $\breve{s}_{k, m}$ and $\breve{n}_{k, m}$ are the projection coefficients for the signal and noise components, respectively. The basis function $\phi_{m}(t)$ is a symbol-long sequence with amplitude $\pm \sqrt{F_{s}^{\prime} / N}$, pulse width $1 / F_{s}^{\prime}$ and sequence length $N=T_{s} F_{s}^{\prime}$ drawn from i.i.d. Bernoulli distribution [9], where $F_{s}^{\prime}$ denotes the "virtual sampling frequency" [10]. Now, stacking the projection coefficients from the $k$ th symbol into vectors $\breve{\mathbf{y}}^{(k)}=\left[\breve{y}_{k, 1}, \breve{y}_{k, 2}, \ldots, \breve{y}_{k, M}\right]^{T}, \breve{\mathbf{s}}=$ $\left[\breve{s}_{k, 1}, \breve{s}_{k, 2}, \ldots, \breve{s}_{k, M}\right]^{T}$ and $\breve{\mathbf{n}}^{(k)}=\left[\breve{n}_{k, 1}, \breve{n}_{k, 2}, \ldots, \breve{n}_{k, M}\right]^{T}$, in CS notation the received vector during the $k$-th symbol becomes

$$
\breve{\mathbf{y}}^{(k)}=\breve{\mathbf{s}}+\breve{\mathbf{n}}^{(k)}=\boldsymbol{\Phi} \mathbf{y}^{(k)}=\boldsymbol{\Phi}\left(\mathbf{s}(\mathbf{\Psi})+\mathbf{n}^{(k)}\right),
$$

where $\mathbf{y}^{(k)} \in \mathbb{R}^{N}$ is the vector of the received signal sampled at $F_{s}^{\prime}, \boldsymbol{\Phi} \in \mathbb{R}^{M \times N}$ is the measurement matrix with each row comprising of virtually sampled $\phi_{m}(t), \mathbf{s} \in \mathbb{R}^{N}$ denotes samples of the desired signal, $\mathbf{n}^{(k)} \in \mathbb{R}^{N}$ is the vector of AWGN samples and $\Psi$ represents the discrete time counterpart of $\psi$. The goal here is to estimate the set of parameters by $M \leq N$ measurements. To continue the derivation, we stack $\breve{\mathbf{y}}^{(k)}$ into $\breve{\mathbf{y}}:=\left[\breve{\mathbf{y}}^{(0) T}, \breve{\mathbf{y}}^{(1) T}, \ldots, \breve{\mathbf{y}}^{(K-1) T}\right]^{T}$ and write the conditional likelihood function as

$$
\begin{gathered}
\Lambda(\boldsymbol{\Psi})=\mathbb{C} \exp \left(-\sum_{k=0}^{K-1}\left(\breve{\mathbf{y}}^{(k)}-\mathbf{\Phi} \mathbf{s}(\boldsymbol{\Psi})\right)^{T} \times\right. \\
\left.\left(2 \sigma_{n}^{2} \boldsymbol{\Phi} \boldsymbol{\Phi}^{T}\right)^{-1}\left(\breve{\mathbf{y}}^{(k)}-\boldsymbol{\Phi} \mathbf{s}(\boldsymbol{\Psi})\right)\right),
\end{gathered}
$$

where $\mathbb{C}$ is a positive constant irrelevant in the formulation, $(\cdot)^{T}$ is the matrix transpose, $(\cdot)^{-1}$ being the matrix inverse, and $\sigma_{n}^{2}$ is the noise sample variance. Because $\boldsymbol{\Phi}$ in (3) is an orthogonal projector, i.e., $\boldsymbol{\Phi} \boldsymbol{\Phi}^{T}=\mathbf{I}_{M}$ [9], the log-likelihood function reduces to

$$
\ln (\Lambda(\boldsymbol{\Psi}))=\sum_{k=0}^{K-1}\left(2 \mathbf{s}^{T}(\mathbf{\Psi}) \boldsymbol{\Phi}^{T} \breve{\mathbf{y}}^{(k)}-\|\mathbf{\Phi} \mathbf{s}(\mathbf{\Psi})\|^{2}\right) .
$$

With $\tilde{\mathbf{x}}$ denoting a trial version of $\mathbf{x}$, the ML estimate to $\Psi$ boils down to

$$
\hat{\mathbf{\Psi}}=\arg \max _{\tilde{\mathbf{\Psi}}}\left\{2 \sum_{k=0}^{K-1} \tilde{\boldsymbol{\Phi}}^{T} \breve{\mathbf{y}}^{(k)}-\sum_{k=0}^{K-1}\|\boldsymbol{\Phi} \mathbf{s}(\tilde{\boldsymbol{\Psi}})\|^{2}\right\},
$$

where $\tilde{\boldsymbol{\Phi}}=\left[\mathbf{s}^{T}(\tilde{\boldsymbol{\Psi}}) \boldsymbol{\Phi}_{1}^{T}, \mathbf{s}^{T}(\tilde{\mathbf{\Psi}}) \boldsymbol{\Phi}_{2}^{T}, \ldots, \mathbf{s}^{T}(\tilde{\boldsymbol{\Psi}}) \boldsymbol{\Phi}_{M}^{T}\right]^{T} \in \mathbb{R}^{M}$ is a vector of coefficients comprising of the inner product between a trial value of $\boldsymbol{\Psi}$ and the $m$-th row of $\boldsymbol{\Phi}$ denoted as $\boldsymbol{\Phi}_{m}$. In the limit as $N \rightarrow \infty$ [12], the argument of (6) becomes the log-likelihood function as

$$
\begin{aligned}
\ln (\Lambda(\tilde{\boldsymbol{\psi}}))= & 2 \sum_{k=0}^{K-1} \sum_{m=1}^{M} \breve{y}_{k, m}^{\prime} \int_{k T_{s}}^{k T_{s}+T_{p r j}} \phi_{m}(t) \hat{s}(t ; \tilde{\boldsymbol{\psi}}) \mathrm{d} t- \\
& \sum_{k=0}^{K-1} \sum_{m=1}^{M}\left(\int_{k T_{s}}^{k T_{s}+T_{p r j}} \hat{s}(t ; \tilde{\boldsymbol{\psi}}) \phi_{m}(t) \mathrm{d} t\right)^{2}, \text { (7) }
\end{aligned}
$$

where the single-symbol trial value of the template is

$$
\hat{s}(t ; \tilde{\boldsymbol{\psi}})=\sum_{l=1}^{L_{c}} \tilde{\alpha}_{l} s\left(t-\tilde{\tau}_{l}-k T_{s}\right),
$$

where $L_{c}$ is the ML parameter denoting the number of strongest taps to be estimated. By the use of (8), we inherently assume the received signal $y(t)$ to be sparse 1 in certain basis within a single symbol, i.e., $L_{c}$ sparse on basis $s(t)$ with arbitrary amplitude and delay in $\tilde{\alpha}_{l}$ and $\tilde{\tau}_{l}$, respectively, for $l=1,2, \ldots, L_{c}$. In (7), $\breve{y}_{k, m}^{\prime}$ is defined as

$$
\breve{y}_{k, m}^{\prime}=\int_{k T_{s}}^{k T_{s}+T_{p r j}} y(t) \phi_{m}(t) \mathrm{d} t, \quad m=1, \ldots, M,
$$

where the parameter $T_{p r j}$ indicates the integration length. Since our system has no inter-frame interference, i.e., $T_{m d s}<$ $T_{f}$, the majority of the received energy is confined within $T_{m d s}$; therefore, we let $T_{p}<T_{p r j} \leq T_{m d s}$. The collection of compressive measurements can be implemented by $M$ integrators, each with an integration interval $T_{p r j}$. In that

\footnotetext{
${ }^{1}$ Although the assumption of sparsity is typically dealt with in the digital domain, one can easily see the resemblance here with the Karhunen-Loéve expansion [12].
} 
case, the sequence length becomes $N=T_{p r j} F_{s}^{\prime}$. Thus, the CS-ML channel estimator reduces to finding the pairs $(\tilde{\boldsymbol{\alpha}}, \tilde{\boldsymbol{\tau}})$ which maximizes (7). Specifically, it comprises of projecting the received signal onto orthornormal basis $\phi_{m}(t)$ for $m=$ $1, \ldots, M$. Hence, instead of sampling at Nyquist rate as in the conventional ML approach, we reduce the sampling rate by collecting fewer compressive measurements.

To continue with the ML derivation, we recognize the compaction of distance 2 , and assume that the spread of multipath energy is confined within the interval $T_{p r j}=\left[0, T_{m d s}\right)$, we rewrite (7) as

$$
\begin{aligned}
\ln (\Lambda(\tilde{\boldsymbol{\psi}})) \cong 2 & \sum_{k=0}^{K-1} \sum_{m=1}^{M} \breve{y}_{k, m}^{\prime} \int_{T_{p r j}} \phi_{m}(t) \hat{s}(t ; \tilde{\boldsymbol{\psi}}) \mathrm{d} t \\
& -\frac{M}{N} \sum_{k=0}^{K-1} \int_{T_{p r j}} \hat{s}^{2}(t ; \tilde{\boldsymbol{\psi}}) \mathrm{d} t .
\end{aligned}
$$

Now, substituting (8) into (10), we obtain

$$
\begin{aligned}
\ln (\check{\Lambda}(\tilde{\psi}))= & 2 \sum_{k=0}^{K-1} \sum_{l=1}^{L_{c}} \tilde{\alpha}_{l} z_{k}\left(\tilde{\tau}_{l}\right)-\frac{M}{N} \sum_{k=0}^{K-1} \sum_{l=1}^{L_{c}} \sum_{l^{*}=1}^{L_{c}} \tilde{\alpha}_{l} \tilde{\alpha}_{l^{*}} \times \\
& \int_{T_{p r j}} s\left(t-\tilde{\tau}_{l}-k T_{s}\right) s\left(t-\tilde{\tau}_{l^{*}}-k T_{s}\right) \mathrm{d} t, \quad(11)
\end{aligned}
$$

where $\ln (\check{\Lambda}(\tilde{\psi}))$ approximates $\ln (\Lambda(\tilde{\boldsymbol{\psi}}))$ after compensating for the compaction of distance, and $z_{k}\left(\tilde{\tau}_{l}\right)$ is defined as

$$
z_{k}\left(\tilde{\tau}_{l}\right)=\sum_{m=1}^{M} \breve{y}_{k, m}^{\prime} \int_{T_{p r j}} \phi_{m}(t) s\left(t-\tilde{\tau}_{l}-k T_{s}\right) \mathrm{d} t .
$$

Assuming there is no correlation between signal echoes within a symbol duration, i.e., $\int_{T_{p r j}} s\left(t-\tilde{\tau}_{l}-k T_{s}\right) s\left(t-\tilde{\tau}_{l^{*}}-\right.$ $\left.k T_{s}\right) \mathrm{d} t=0$ for $l^{*} \neq l$, (11) reduces to

$$
\ln (\check{\Lambda}(\tilde{\boldsymbol{\psi}}))=2 \sum_{k=0}^{K-1} \sum_{l=1}^{L_{c}} \tilde{\alpha}_{l} z_{k}\left(\tilde{\tau}_{l}\right)-\frac{K M E_{s}}{N} \sum_{l=1}^{L_{c}} \tilde{\alpha}_{l}^{2},
$$

where $E_{s}=\int_{T_{p r j}} s^{2}(t) \mathrm{d} t$ is the symbol energy.

We can now estimate the parameter $\boldsymbol{\psi}$ by looking for the pair $(\boldsymbol{\alpha}, \boldsymbol{\tau})$ which minimizes (13). The minimization is carried out first by keeping $\tau$ fixed and varying $\boldsymbol{\alpha}$. Specifically, differentiating (13) with respect to $\tilde{\alpha}_{l}$ and equating it to zero, we get

$$
\tilde{\alpha}_{l}=\frac{N}{K M E_{s}} \sum_{k=0}^{K-1} z_{k}\left(\tilde{\tau}_{l}\right) .
$$

Substituting (14) into (13), the optimization problem simplifies to

$$
\hat{\tau}_{l}=\arg \max _{\tilde{\tau}_{l}}\left(\sum_{k=0}^{K-1} z_{k}\left(\tilde{\tau}_{l}\right)\right)^{2}, \quad l=1, \ldots, L_{c},
$$

\footnotetext{
${ }^{2}$ In CS theory, for any $\mathbf{x} \in \mathbb{R}^{N}$, the random variable $\|\mathbf{\Phi} \mathbf{x}\|^{2}$ has an expected value $(M / N)\|\mathbf{x}\|^{2}[9]$. Likewise, in our case $\|\mathbf{\Phi} \mathbf{S}(\boldsymbol{\Psi})\|^{2}$ has an expected distance of $(M / N)\|\mathbf{s}(\mathbf{\Psi})\|^{2}$.
}

and, the gains are estimated as

$$
\hat{\alpha}_{l}=\frac{N}{K M E_{s}} \sum_{k=0}^{K-1} z_{k}\left(\hat{\tau}_{l}\right), \quad l=1, \ldots, L_{c} .
$$

Comparing to the conventional ML formulation of the path delays and gains [4], the CS-ML channel estimator takes on the extra step of projecting the received signal onto the $M$ independent Bernoulli sequences for compression; thereafter, the correlation between the compressed signal and the compressed pulse template at each possible path delay. Although the compression of a signal results in a reduction of the expected distance by $\sqrt{M / N}$ [9], it can be compensated by scaling the gain estimates accordingly, as shown in the derivation. Altogether, the redundancy in signal processing guarantees a reduced sampling rate, a necessity especially to alleviate the burden of acquiring fine-scaled samples in the ML scheme. Comparing to MP, which relies heavily on the selection of design parameters that are subject to change depending on the environment, our approach uses only the traditional ML parameters. Moreover, unlike the MP technique that requires a complete $N \times N$ orthonormal basis set as the dictionary for accurate estimation, the resolution of the CS-ML depends entirely on how the maximization is performed. Finally, we stress that, whereas only an isolated pulse is transmitted and its incurring multipath recorded for processing with MP [11], the CS-ML capitalizes on the noise statistics formulation of ML to achieve a reliable performance.

\section{IMPLEMENTATION ISSUES}

To compare the implementation issues, we resort to the digital domain. Specifically, after passing $y(t)$ through a lowpass filter of bandwidth $B$ and assuming no signal distortion, we collect $N^{\prime}=T_{s} / T_{s a}$ integer samples per symbol at the Nyquist rate $1 / T_{s a}=2 B$ from the filter output $r(t)$. We digitize the delay trial value as $\left\lfloor\tilde{\tau}_{l} / T_{s a}\right\rfloor:=\left(l_{p} Z+l_{f}\right)$, where $Z:=\left\lfloor T_{p} / T_{s a}\right\rfloor$ denotes the integer number of samples per pulse, $\lfloor\cdot\rfloor$ being the flooring function, and $l_{p}$ and $l_{f}$ are the pulse delay and fine sample delay indexes, respectively, then, $r[i]:=r\left(i T_{s a}\right)$ and $s\left[i-k N^{\prime}-\left(l_{p} Z+l_{f}\right)\right]:=s\left(\left(i-k N^{\prime}-\right.\right.$ $\left.\left.\left(l_{p} Z+l_{f}\right)\right) T_{s a}\right)$. With these notations, the delay estimates of the ML estimator operating digitally with $l_{p}$-space estimator, i.e., $l_{f}=0$, on the $l_{p}$-th path can be written as

$$
\begin{aligned}
\hat{l}_{p} & =\arg \max _{\tilde{l}_{p}}\left(\sum_{k=0}^{K-1} v_{k}\left[\tilde{l}_{p}\right]\right)^{2}, \\
v_{k}\left[l_{p}\right] & =\sum_{i=1}^{K N^{\prime}} r[i] s\left[i-k N^{\prime}-l_{p} Z\right],
\end{aligned}
$$

where we have approximated the integral by a summation. Similarly, the digital representation of CS-ML is

$$
\begin{aligned}
\hat{l}_{p} & =\underset{\tilde{l}_{p}}{\arg \max _{p}}\left(\sum_{k=0}^{K-1} z_{k}\left[\tilde{l}_{p}\right]\right)^{2}, \\
z_{k}\left[l_{p}\right] & =\sum_{m=k}^{(k+1) M} \breve{r}^{\prime}[m] \breve{s}^{\prime}\left[m, l_{p}\right],
\end{aligned}
$$




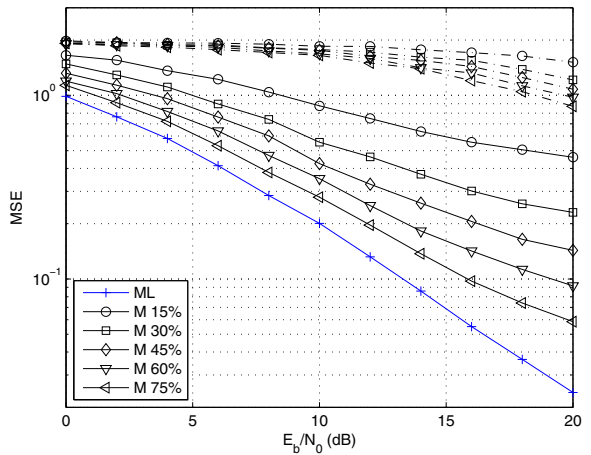

Fig. 1. MSE as SNR varies for $K=20, T_{p r j}=T_{f}$ and $L_{c}=20$. The solid and dash-dot lines represent CS-ML and MP channel estimators, respectively.

where $\breve{s}^{\prime}\left[m, l_{p}\right]:=\sum_{i=k}^{(k+1) N^{\prime}} \phi_{m}[i] s\left[i-k N^{\prime}-l_{p} Z\right]$ is the coefficient of the discretized template after being projected onto the $m$-th basis for $m=1, \ldots, M$. Therefore, comparing both (18) and (20), we see that compressive measurements essentially reduce the Nyquist rate by a factor of $N^{\prime} / M$. This reduction amounts to extra savings in hardware and is indispensable if we are to implement the asymptotic ML estimator.

\section{Simulation Results}

We examine the performance of the channel estimators by computer simulation. Unless stated otherwise, $T_{f}=100 \mathrm{~ns}$, $N_{f}=20$, and $s(t)$ is modeled as a Gaussian doublet with $T_{p}=2 \mathrm{~ns}$. To accurately portray the UWB channel, we use the IEEE 802.15.3a CM1 channel model of [13], which depicts a line-of-sight environment with $T_{m d s}=60 \mathrm{~ns}$. We evaluate the performance of these estimators in terms of the reconstruction mean squared error (MSE) and the bit-error rate (BER) of a Rake receiver under a varying SNR.

\section{A. MSE Performance}

We compare all three channel estimators when $T_{p r j}=T_{f}$ and in a resolvable channel, i.e., $\left|\tau_{i}-\tau_{j}\right| \geq T_{p}$. In the case when pulse overlapping is allowed, we show in the next section that the CS-ML still achieves performance equivalent to the ML but at a reduction in complexity. Fig.1 1illustrates the MSE characteristic as SNR varies for $K=20$, and $L_{c}=20$. As shown, MP results in the worst performance since its estimates are based on the multipath returns of an isolated pulse. In contrast, the gain of employing DA estimation is quite obvious with the conventional ML giving the best performance while the CS-ML falling in between ML and MP. Depending on the design parameter $M$ (viewed here as a percentage of $N$ ), the performance of CS-ML varies due to a varying compression rate, with a small $M$ indicating high compaction and lower complexity, but more error prone. Therefore, the parameter $M$ must be chosen with care.

The primary advantage of the ML scheme is to rely on an increasing number of pilot symbols to enhance the overall

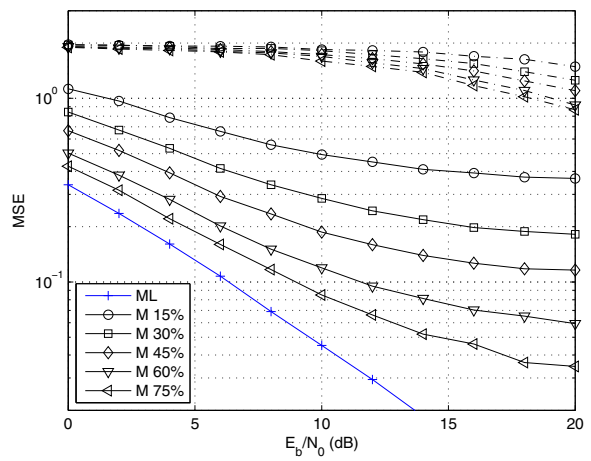

Fig. 2. MSE as SNR varies for $K=100, T_{p r j}=T_{f}$ and $L_{c}=20$. The CS-ML and MP channel estimators are denoted by solid and dash-dot lines, respectively.

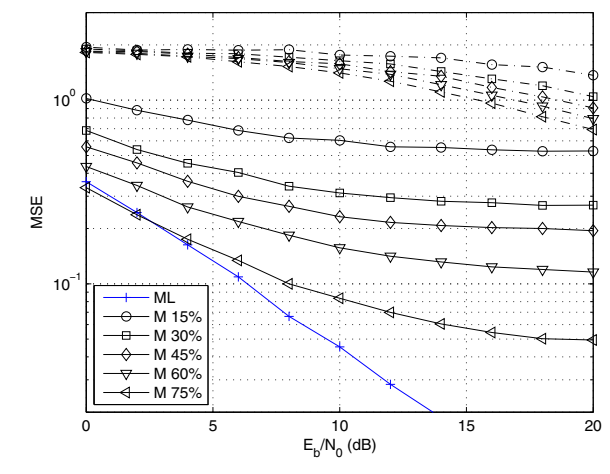

Fig. 3. MSE as SNR varies for $K=100, T_{p r j}=40 \mathrm{~ns}$ and $L_{c}=20$. The CS-ML and MP channel estimators are denoted by solid and dash-dot lines, respectively.

estimator performance. To demonstrate this, Fig. 2] plots MSE versus SNR for $K=100$. Comparing to when $K=20$, we see an improvement for both ML and CS-ML estimators, especially at low SNR. Unfortunately, MP cannot take the advantage of elongating the observation window as it is based on the processing of an isolated pulse. The reduction in complexity can go even further for the CS-ML by reducing $T_{p r j}$ in (9). Whereas this has not been done for the conventional ML scheme, the gains with a varying $T_{p r j}$ in CS-ML are twofold: first, by decreasing the integration region we are reducing the amount of noise in the system and, with a $T_{p r j}$ properly chosen, the performance may substantially improve. Secondly, reducing $T_{p r j}$ implicitly reduces the number of integrators necessary for compression, resulting in an overall reduction on the system complexity. We illustrate the effect of reducing $T_{p r j}$ in Fig. 3 which plots the MSE as SNR varies for $T_{p r j}=40$ ns. As expected, decreasing $T_{p r j}$ improves the performance at low SNR but hinders the accuracy at high SNR where a higher floor is clearly visible. Fortunately, this flooring behavior does not diminish the BER performance, as is shown next. 


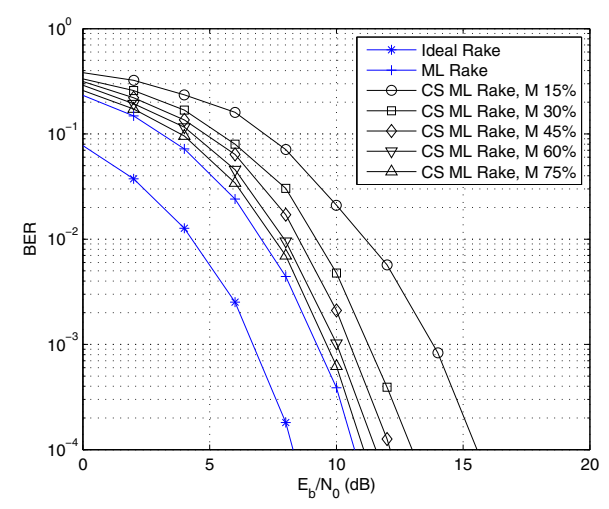

Fig. 4. BER of A-PAM Rake receiver for $K=20$ and $L_{c}=20$. For CS-ML, we let $T_{p r j}=40 \mathrm{~ns}$ and $M$ is varied for different compression rate.

\section{B. BER Performance}

To evaluate the BER performance, we consider the reception of antipodal pulse amplitude modulation (A-PAM) with a Rake receiver in a single-user scenario with overlapping pulses, i.e., $\left|\tau_{i}-\tau_{j}\right|<T_{p}$. The system first sends $K$ pilot symbols for DA channel estimation prior to the burst of data transmission. Based on the sequences of estimated multipath gains and delays, i.e., $\left\{\hat{\alpha}_{l}\right\}_{l=1}^{L_{c}}$ and $\left\{\hat{\tau}_{l}\right\}_{l=1}^{L_{c}}$, respectively, from either the CS-ML or ML estimators the Rake receiver then performs the maximum-ratio combining [4] with $T_{p r j} \in\left[0, T_{f}\right)$ as the integration interval on the $j$-th frame to produce the decision statistic. Fig. 4 illustrates the BER performance of the two schemes with $K=20, L_{c}=20$ and $T_{p r j}=40 \mathrm{~ns}$. For the CS-ML, $M$ is allowed to take on different values, representing different compression rate. The data burst is set to 1,000 bits and a thousand channels are averaged. As shown, the estimation capability of the CS-ML can be as good as the ML but at a reduced sampling rate, e.g., with $45 \% \leq M \leq 75 \%$ the performance is almost as good as and sometimes very close to the ML. Apart from varying $M$, the distinct advantage of ML is to rely on elongating the observation window to boost the performance in a noisy environment. To show that, Fig. 5 graphs the BER performance for $K=100$. Comparing to when $K=20$, we see an improvement at all values of $M$ for CS-ML, and the performance of both estimators approaching that of the ideal Rake. In summary, although the CS-ML demonstrates a superior performance as $M$ and $K$ increase, one would still need to consider the complexity tradeoff and with a larger $M$ incurring a higher complexity, i.e., a larger number of intergrators.

\section{CONCLUSION}

The rich multipath characteristic of UWB channel motivates the use of Rake receivers. Although DA ML channel estimator shows a promising performance, the Nyquist criterion renders its implementation difficult. In this paper, we have proposed a CS-ML channel estimator which combines the compression framework of CS for sampling rate reduction while retaining

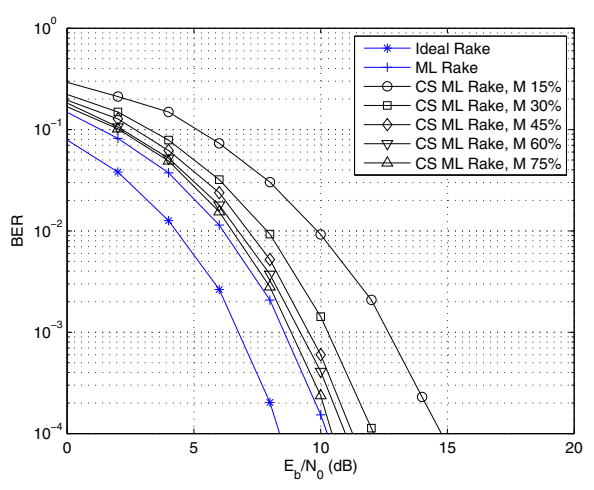

Fig. 5. BER of A-PAM Rake receiver for $K=100, L_{c}=20$ and $T_{p r j}=$ $40 \mathrm{~ns}$.

the noise statistics formulation of ML to achieve a reliable performance. From simulation, we have shown that with far fewer measurements the performance of our scheme supersedes that of MP-based estimator and can be as good as the conventional ML estimator, but with a trade off in complexity.

\section{ACKNOWLEDGMENT}

This work was supported by the Natural Sciences and Engineering Research Council (NSERC) of Canada.

\section{REFERENCES}

[1] L. Yang and G. Giannakis, "Ultra-wideband communications: an idea whose time has come," IEEE Signal Process. Mag., vol. 21, no. 6, pp. 26-54, 2004.

[2] C. Carbonelli and U. Mitra, "Clustered ML Channel Estimation for Ultra-Wideband Signals," IEEE Trans. Wireless Commun., vol. 6, no. 7, pp. 2412-2416, 2007.

[3] M. Win and R. Scholtz, "Characterization of ultra-wide bandwidth wireless indoor channels: a communication-theoretic view," IEEE J. Sel. Areas Commun., vol. 20, no. 9, pp. 1613-1627, 2002.

[4] V. Lottici, A. D'Andrea, and U. Mengali, "Channel Estimation for UltraWideband Communications," IEEE J. Sel. Areas Commun., vol. 20, no. 9, pp. 1638-1644, 2002.

[5] Z. Tian and V. Lottici, "Low-complexity ML timing acquisition for UWB communications in dense multipath channels," IEEE Trans. Wireless Commun., vol. 4, no. 6, pp. 3031-3038, 2005.

[6] C. Carbonelli and U. Mengali, "Synchronization Algorithms for UWB Signals," IEEE Trans. Commun., vol. 54, no. 2, pp. 329-338, 2006.

[7] J. Kusuma, I. Maravic, and M. Vetterli, "Sampling with finite rate of innovation: channel and timing estimation for UWB and GPS," in Proc. of IEEE ICC, vol. 5, May 2003, pp. 3540-3544.

[8] E. Candes, J. Romberg, and T. Tao, "Robust uncertainty principles: exact signal reconstruction from highly incomplete frequency information," IEEE Trans. Inf. Theory, vol. 52, no. 2, pp. 489-509, 2006.

[9] M. Davenport, M. Wakin, and R. Baraniuk, "Detection and estimation with compressive measurements," Dept. of ECE, Rice University, Tech. Rep., 2006.

[10] Z. Wang, G. Arce, B. Sadler, J. Paredes, and X. Ma, "Compressed Detection for Pilot Assisted Ultra-Wideband Impulse Radio," in Proc. of IEEE ICUWB, Sept. 2007, pp. 393-398.

[11] J. Paredes, G. Arce, and Z. Wang, "Ultra-Wideband Compressed Sensing: Channel Estimation," IEEE J. Sel. Topics Signal Process., vol. 1, no. 3, pp. 383-395, 2007.

[12] H. Van Trees, Detection, Estimation, and Modulation Theory. Part I. New York: John Wiley \& Sons, 1968.

[13] A. Molisch, J. Foerster, and M. Pendergrass, "Channel models for ultrawideband personal area networks," IEEE Wireless Commun. Mag., vol. 10, no. 6, pp. 14-21, 2003. 